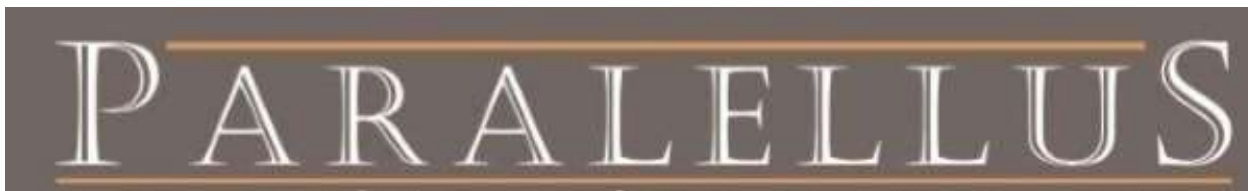

REVISTA ELETRÔNICA EM CIÊNCIAS DA RELIGIÃO - UNICAP

Dossiê

(D)

Volume 8 - Número 18

O CRISTIANISMO E AS SUAS REFORMAS II

IL CRISTIANESIMO E LE SUE RIFORME II

doi: 10.25247/paralellus.2017.v8n18.p237-250

\title{
OLTRE LE BARRIERE CONFESSIONALI: VITA MONASTICA E UNITÀ DELLA CHIESA
}

Mariella Carpinello*

\section{RIASSUNTO}

La comunità di Taizè ha giocato un ruolo fondamentale nella promozione del dialogo ecumenico della seconda metà del XX secolo, attraverso una serie di contatti personali; il saggio presenta, attraverso la citazione di documenti inediti, una parte di questi contatti, prima del Concilio Vaticano II, tra la comunità di Taizè, papa Pio XII e Grottaferrata.

Parole chiavi: Taizè. Ecumenismo. Spiritualità. Pio XII.

\section{ABSTRACT}

The community of Taizè played very relevant role for the promoting the ecumenical dialogue in the second half of the XXth century, by several personal relationships. The essay deals with some of this kind of personal relationships, before the Vatican II, among the community of Taizè, pope Pus XII, Grottaferrata, quoting inedited sources.

Keywords: Taizè. Ecumenism. Spirituality. Pius XII.

\section{INTRODUZIONE}

II 24 marzo 1966 l'incontro fra Paolo VI e il primate anglicano Michael Ramsey apre un nuovo corso ai rapporti fra Chiesa cattolica e anglicanesimo, ponendo le premesse

\footnotetext{
* Docente di ecumenismo presso il Claretianum, Istituto di Teologia della Vita Consacrata, della Pontificia Università Lateranense, di Roma. Ha pubblicato saggi sulla storia della comunità monastica di Grottaferrata e sulla spiritualità ecumenica. Link: http://www.claretianum.org/professori.php?a=1\&id=644. E-mail: mariellacarpinello@tin.it. 
alla costituzione della Anglican -Roman Catholic International Commission (1967) per il regolare confronto fra le parti.

Alcuni importanti antefatti dell'evento risalgono al settembre 1947, quando un esponente dell'anglicanesimo romanizzante è ricevuto in Vaticano da mons. Montini, al tempo pro segretario della Segreteria di Stato. L'iniziativa di quell'incontro, avvenuto in una stagione a dir poco arretrata per gli scambi ecumenici, è partita da madre Maria Pia Gullini (1892-1959)1', abbadessa della trappa di Grottaferrata, ove nel 1939 la venticinquenne sr. M. Gabriella Sagheddu è morta dopo aver offerto la vita per l'unità dei cristiani. Una biografia ${ }^{2}$ della giovane ne ha diffuso la fama nel movimento ecumenico internazionale, l'apostolo dell'unità Paul Couturier ne ha propugnato il modello e nel suo nome s'è stretta un'amicizia fra la comunità di Grottaferrata e quella dei monaci di Nashdom, anglicani osservanti la Regola benedettina. II vivissimo senso della Chiesa che anima il suo abbaziato ha suggerito alla Gullini un audace progetto: invitare a Grottaferrata il maestro dei novizi di Nashdom, dom Benedict Ley, per fargli conoscere quel mondo cattolico da cui già è attratto tramite san Benedetto. Al fine d'introdurlo in Vaticano, ha coinvolto Igino Giordani, politico e pubblicista, Felice Beretta, segretario generale dell'Unione Missionaria del Clero, Giulio Penitenti, la cui opera per l'unione ha visto la luce a Roma da pochi anni, Charles Boyer, docente alla Pontificia Università Gregoriana e presidente della società "Unitas", Vincenzo Ceresi (1869-1958) e Paolo Caresana (1882-1973), questi ultimi vicinissimi a colui che per apertura di spirito e d'intelletto può garantire successo all'iniziativa: Giovanni Battista Montini.

\footnotetext{
1 E. FRANCIA, Lettere e scritti di Madre Pia, Editrice "Messa degli artisti", Roma, 1971 M A TESCARI, Madre Pia Gullini, una grande badessa del XX secolo, Velar, 2009; ID. Madre Pia Gullini, una figura profetica del monachesimo italiano del XX secolo Cantagalli, 2016; M. CARPINELLO, Monachesimo femminile e profezia, l'azione ecumenica di Madre Maria Pia Gullini, Cantagalli, 2015; quest'ultimo volume riporta in forma estesa documenti d'archivio conservati a Nostra Signora di S. Giuseppe di Vitorchiano che nel presente articolo appaiono in forma abbreviata.

2 M. G. DORE; I. GIORDANI, Suor Maria Gabriella (1914-1939): dalla Trappa per l'unità della Chiesa. Brescia, 1940. Altri studi: C. TESTORE Suor Maria Gabriella Trappista - 1914-1939, Vitorchiano 1958; P.BELTRAME QUATTROCCHI, La Beata Maria Gabriella dell'Unità, Vitorchiano, 1980; M.DELLA VOLPE, La strada della gratitudine - Suor Maria Gabriella, Milano, 1983; B. MARTELET, Una vita per l'unità dei cristiani, Suor Maria Gabriella, Roma 1987 GABRIELLA DELL' UNITÀ (Beata Maria Gabriella Sagheddu), Lettere dalla Trappa, a cura di M. CARPINELLO, Cinisello Balsano, 2006 D. SPANU Temi di vita spirituale nelle lettere della Beata Maria Gabriella Sagheddu, Cagliari, 2007: ID. Artefice di unità: profilo spirituale della beata Maria Gabriella Sagheddu. Firenze, 2009.ID. Beata Maria Gabriella Sagheddu, testimone credibile del Vangelo di unità, Cagliari, 2014.
} 
Si è giunti così al 22 settembre 1947, quando, in un clima ancor cauto, ma molto disponibile, Montini accoglie in Vaticano l'ospite inglese ${ }^{3}$. Dopo avere premesso che nell'immediato non intende passare al cattolicesimo, onde non destabilizzare il generale processo di confronto fra Chiese, dom Ley assicura di lavorare per dare una spiritualità benedettina alle numerose comunità religiose di area anglicana delle quali ha cura. Quindi consegna a monsignore - che condivide le ragioni della sua prudenza - una relazione intitolata Catholicity, stilata a Nashdom in risposta a progetti di unificazione fra anglicani e nonconformisti. Montini desidererebbe ricevere anche un rapporto sull'attuale situazione fra anglicani e cattolici e dom Ley s'impegna ad inviarglielo, quindi precisa che la comunità di Nashdom non chiede nulla al papa, ma spera in una maggiore chiarificazione della posizione reciproca ed è pronta alla riordinazione ${ }^{4}$. Montini assicura che la questione è in corso di studio e che il dialogo appena iniziato avrà conseguenze.

Il giorno seguente dom Benedict gli scrive da Grottaferrata per garantirgli che testimonierà presso i confratelli anglicani la carità dei cattolici di Roma e per meglio chiarire alcuni aspetti già trattati a voce ${ }^{5}$ :

Se i nostri vescovi accettassero questa comunione nella sua forma attuale, la Comunità Anglicana si scinderebbe. Le comunità religiose femminili e maschili della Chiesa d'Inghilterra sarebbero alla testa di questa scissione. Perché certamente la vita religiosa non potrebbe prosperare in un corpo che non accetta il principio della Rivelazione Divina e fa della religione il frutto di uno stato d'animo mutevole e soggettivo.

La mia Comunità (Nashdom Abbey, Burnham, Bucks) esercita grande influenza sulle altre. Noi usiamo (ed altre quattro comunità con noi) il Messale Romano, recitiamo l'Ufficio Benedettino in latino, e seguiamo l'antica spiritualità benedettina; siamo perciò in grado di dare, a coloro che si affidano spiritualmente alle nostre cure, la vera direzione soprannaturale nella vita religiosa. leri ho scritto in Inghilterra per chiedere che Le siano inviate le ultime pubblicazioni anglicane circa i nostri Ordini, e spero che giungano a Sua Eccellenza fra qualche settimana. Manderò un rapporto sull'attitudine dei cattolici in Inghilterra verso gli acattolici, subito dopo il mio ritorno. Sarei molto contento di

${ }^{3}$ Romilda Tadini, amica della trappa presente all'incontro, scrive un rapporto intitolato Ecumenismo preconciliare. Appunti -ricordo. 18-25 settembre 1947; altro rapporto è firmato da suor Fara: Visita di dom Benedict Ley di Nashdom Abbey alla Trappa di Grottaferrata - 18-25 settembre 1947. Entrambi i documenti sono conservati nell'archivio del monastero di Vitorchiano (d'ora in poi AV).

${ }^{4}$ Leone XIII aveva invalidato le ordinazioni anglicane nel 1896 con bolla Apostolicae Curae.

5 || 23.9.1947. 
dare a Sua Eccellenza qualsiasi informazione che Ella potesse desiderare ${ }^{6}$.

Notiamo il ruolo assegnato alla vita consacrata - tramite per il quale la Chiesa romana è in contatto carismatico con la Chiesa anglicana - nell'impedire che i cristiani separati si estranino dai dogmi cattolici.

Dopo l'attesissima udienza con Pio XII, felice per essersi avvicinato al centro del cattolicesimo quanto gli sarebbe sembrato impensabile solo pochi giorni prima, dom Benedict riparte. II bilancio del viaggio italiano includerà un esame di coscienza?:

In Inghilterra è l'antipatia atavica verso Roma che rende tanta gente (del resto ottima) ancora attaccata a pregiudizi e sospetti. Così se noi dovremo uscire dalla Chiesa d'Inghilterra ci vorrà del tempo per portare quelli che sono con noi a vedere Pietro nel modo giusto.

Lo stesso vale per l'amica della trappa Romilda Tadini, che ha accompagnato dom Ley nelle sue giornate italiane ${ }^{8}$ :

C'è chi pensa che l'anelito all'unità di questa massa immensa di separati sia un lievito religioso assai potente. Si oserebbe dire che i cattolici - almeno una gran parte dei laici - godano in pace, in un beato egoismo, l'unità insorpassabile che la Chiesa di Roma conserva in sé e loro comunica, senza chiedersi altro. E questo stato d'animo scandalizza a ragione i separati, quando si accorgono di tale situazione, perché essi vivono in un punto di vista totalmente diverso. Essi vivono l'unità come sinonimo di santità, nel discorso di Gesù nell'Ultima Cena, sintesi di tutto il comando evangelico e non solo come nota della Dottrina e del Governo della vera Chiesa. Essi perciò non sanno quale lezione di catechismo danno a noi, fedeli cattolici, quando, con una semplicità che commuove, credono di trovare in noi la perfezione dell'unità; anzi (per adoperare le loro parole) la sintesi dell'unità, che è il segno indiscutibile dell'appartenenza a Cristo. I separati realizzano assai più di noi la preghiera di Gesù "che essi siano uno", avvertendo con una carità pratica vivissima il valore dell'altro, ciò che a volte li fa più forti di noi cattolici ... Essi sono "uno" nell'azione sociale assai più di noi.

Ci siamo più o meno accorti che tutto il mondo guarda al Papa come simbolo di un'unità superconfessionale; ma, più di noi, se ne sono accorti i separati. L'idea dell'unità della legge morale, la teologia

${ }^{6}$ La risposta data 9.12.1948: Mio caro Amico, desidero accusarle ricevuta della sua gentile lettera del 23 settembre e ringraziarla della sua cortesia e considerazione nel darmi le sue impressioni personali sulla recente Conferenza di Lambeth. Assicurandola del mio continuato ricordo nelle mie quotidiane preghiere per l'unione di tutti i Cristiani nell'unica vera Chiesa, io rimango sinceramente suo in Xto.GB Montini. AV.

${ }^{7}$ A. M. Gullini, 6 .6. 1948.

8 Vedi nota 5. 
morale di autorità è una delle attrattive, anzi l'attrattiva suprema dei separati e di tutti gli a-religiosi verso l'autorità del Papa e prova implicita dell'autorità di Cristo in lui. Noi cattolici non ci accorgiamo come le questioni inerenti a divorzio e matrimonio, limitazione delle nascite, matrimonio d'esperimento, aborto, eutanasia, suicidio, sono importanti in vista della salvezza della dignità umana anche dal punto di vista naturale, oltre il quale non vi sarebbe nessuna differenza tra la vita animale e la vita umana ... Ora, lontano da Roma, questa voce che si eleva con chiarezza adamantina a sentenziare con obiettiva sicurezza su questioni terribili, con evidente capacità di essere ubbidita, se si vuole la salvezza del genere umano, sta stabilendo una corrente papale che avrà conseguenze mondiali.

Nel viaggio di ritorno verso l'Inghilterra, dom Ley fa tappa a Lione presso Paul Couturier. Informato di ciò che è accaduto, l'abbé inizia ad operare affinché altri fratelli separati entrino in rapporto diretto con la Santa Sede ${ }^{9}$. L'11 febbraio 1948 scrive a Charles Boyer per assicurarsi di poter contare su di lui. Nell'ottobre dello stesso anno consiglia al teologo anglicano Geoffrey Curtis di affidare al cardinale Gerlier, in partenza per Roma, una memoria sulla posizione dell'anglicanesimo in fronte alla Santa Sede. Scritta secondo il principio cor ad cor loquitur, motto cardinalizio di John Henri Newman, il documento informa che molti anglicani impegnati per l'unità nella Church of England attendono indicazioni da parte di Roma e pone alcuni quesiti essenziali ${ }^{10}$ :

Roma è sufficientemente informata delle cose anglicane? Sa che 3.000 preti anglicani pregano per il Papa? Non sarebbe bene che questa informazione le giungesse dagli anglicani stessi e in tal caso che siano approvati? Di contro il Vaticano accetterebbe d'indirizzare le sue encicliche via posta ai fratelli separati, dato che è sempre difficile procurarsele per intermediario?

La memoria suscita grande interesse in Pio XII.

II 9 agosto del '49 dom Benedict scrive a madre Pia:

L'Abbé Couturier è convinto che Padre Curtis dovrebbe dare un seguito a questa lettera e all'effetto che essa ha avuto sul Santo Padre e dovrebbe recarsi a Roma. Ho potuto capire che Mons. Montini ha trovato l'alloggio per lui nell'Abbazia di S. Girolamo a Roma.

\footnotetext{
9 MAURICE VILLAIN in L'Abbé Paul Couturier, apôtre de l'Unité chrétienne, Paris-Tournai, 1957, p. 237 e seguenti.

10 ID.p. 238.
} 
Il viaggio in Italia di padre Curtis evidenzia un avanzamento netto, se il pro segretario dello Stato Vaticano si cura della sua ospitalità ${ }^{11}$ e se papa Pio XII anticipa il visitatore, chiedendo di conoscerne preventivamente le argomentazioni.

II 4 novembre del '49 dom Benedict informa madre Pia d'aver domandato a mons. Montini udienza per un altro esponente anglicano:

II Dott. Prestige ${ }^{12}$ ha nella Chiesa d'Inghilterra una posizione non dissimile da quella di Mons. Montini. E' segretario del Consiglio della Chiesa Anglicana per le relazioni con l'Estero. Egli non è né della Chiesa Alta né della Bassa, ciò che noi chiamiamo centrale. Non è molto comprensivo della posizione cattolica, ma io so che non potrà fare a meno di vedere cosa sia la carità cattolica - cioè la santità cattolica - e che cosa essa significhi se verrà a farvi una visita ${ }^{13}$.

La serie di scambi inauguratasi nel 1947 consegue un progresso a dir poco significativo quando, il 15 marzo 1949, ad approdare alla Segreteria di Stato Vaticana sono due protestanti, Roger Schutz e Max Thurian. Ricordiamo che, proprio nella pasqua dello stesso anno, i primi fratelli di Taizé pronunciano i voti monastici, entrando in comunione con il cattolicesimo senza rompere con le proprie origini confessionali. Forse è dal 1521, anno di pubblicazione del De votis monasticis iudicium di Martin Lutero, che la teologia riformata non ha un'occasione tanto concreta d'inoltrarsi nella questione del rapporto battesimo - vita monastica ${ }^{14}$.

Prima di considerare l'incontro fra Montini e i fondatori di Taizé, dedichiamo qualche parola alla situazione generale nella quale avviene.

Negli stessi mesi la Santa Sede è in una situazione spinosa in fronte al movimento ecumenico. II 23 agosto 1948 la conferenza di Amsterdam ha dato vita al Consiglio Ecumenico delle Chiese. Prefigurato nel 1937 dalla conferenza di Edimburgo e non conseguito a motivo della guerra, il traguardo è infine stato raggiunto con la partecipazione delle Chiese ortodosse (tranne quelle impedite da coercizioni politiche)

\footnotetext{
${ }_{11}$ Nel settembre 1949 Curtis scrive a Couturier: «La dolcezza e la bontà dei benedettini francesi presso i quali mi trovo è insuperabile. Non esistono ospiti più incantevoli e compiti».

${ }^{12}$ George Leonard Prestige (1889-1955), teologo anglicano, autore di Dio nel pensiero dei Padri (1952).

${ }_{13}$ Prestige, come già ha fatto Curtis, visiterà infatti la trappa di Grottaferrata.

14 Rapporto cui già l'antico monachesimo dedicò energica elaborazione dottrinale: A. de Vogüé II monastero e la Chiesa, in La regola di S. Benedetto. Commento dottrinale e spirituale, Padova, 1984, pp. 25-73.
} 
e di quasi tutte le confessioni cristiane. Praticamente l'unica grande assente risulta Roma. Con il monito Compertum est (5 giugno 1948), infatti, la Santa Sede ha vietato sia ai laici che agli ecclesiastici di partecipare ai lavori di Amsterdam, mostrando un irrigidimento rispetto alla conferenza di Edimburgo, alla quale erano comparsi inviati cattolici, e provocando una generale esigenza di chiarimento ${ }^{15}$. Pochi mesi dopo Max Thurian ha scritto un equo commento al Compertum est ${ }^{16}$, sostenendo che esso è un richiamo alla disciplina e non un'intimidazione. L'abbé Couturier ne ha poi inviato copie al Santo Padre e a mons. Montini, sperando di sollecitare le attese spiegazioni.

Ora, ricevendo in udienza i fondatori di Taizé, Montini conferma il carattere di misura disciplinare interna del Compertum est e, poiché Roma non è stata ufficialmente invitata a mandare rappresentanti ad Amsterdam, chiede che si studi una formula d'inviti in vista delle prossime assemblee. Domanda inoltre che sia riconosciuta la posizione della Chiesa romana, obbligata a mantenersi fedele al fine che Cristo le ha assegnato: essere la sola Chiesa di Cristo, il solo corpo mistico di Cristo, la sola Sposa di Cristo, pertanto impedita ad allinearsi ad altre Chiese per ricercare un'unità della quale possiede il principio. Gli stessi temi vengono ripresi il 16 marzo durante l'udienza concessa a da Pio XII Schutz e Thurian, con vivissima soddisfazione di entrambi.

Entro lo scadere di un anno, il 28 febbraio 1950, il Sant'Ufficio emette l'istruzione Ecclesia Catholica ${ }^{17}$ e per la prima volta apre spiragli all'ecumenismo, presentandolo come frutto dell'ispirazione dello Spirito Santo. Rispetto al Compertum est, il nuovo documento è più favorevole agli sforzi comuni, cui potranno partecipare in forma non ufficiale teologi designati dalla Santa Sede, che si riserva il diritto di sceglierli ed esserne unica mandataria.

II più stretto collaboratore di Couturier, Maurice Villain, osserva al riguardo ${ }^{18}$ :

Dopo la conferenza di Amsterdam, i cui atti li avevano molto impressionati, alcuni teologi romani si accorsero che la questione

\footnotetext{
15 Interrogativi sulla posizione della Santa Sede compaiono in alcuni articoli del Times: il primo s'intitola Catholicism to day (31 ottobre 1949), l'ultimo Rome and Europe (29 novembre 1949); rilevante quello del pastore Marc Boegner in Le Figaro dell'11 gennaio 1950.

${ }^{16}$ A propos de l' avertissement du Saint-Office du juin 1948, in "Verbum Caro", dicembre 1948, pp. 184189.

17 Emessa il 20 dicembre 1949.

18 L'abbé Paul Couturier, p. 246.
} 
ecumenica cominciava a prendere un orientamento - se non una consistenza - dogmatica, a cui non era estraneo il moltiplicarsi dei contatti tra protestanti e cattolici. Se dunque il pensiero cattolico iniziava ad essere presente al movimento ecumenico, non era opportuno riconoscerlo e regolamentarlo? Ma fino a quale punto le rispettose osservazioni dei fratelli di Taizé a proposito del monitum e le loro istanze nel chiedere una spiegazione ufficiale accelerarono la comparsa della istruzione Ecclesia Catholica?

Tra il 19 e il 25 giugno 1950 Thurian e Schutz sono di nuovo in Italia in udienza dal papa e negli stessi giorni visitano Grottaferrata accompagnati da Amélie, madre di frère Roger. Fra la Gullini e Amélie nasce un'amicizia, testimoniata a partire dal 1957 dalle lettere che restano della loro corrispondenza ${ }^{19}$. Amélie, il cui ruolo nelle origini di Taizé è ben noto, indica in madre Gullini la prima religiosa con la quale Taizé abbia stretto vincoli di preghiera ecumenica ${ }^{20}$.

La visita che facemmo alla Trappa di Grottaferrata è rimasta per me indimenticabile! Ne parlo spesso, e pure Fr. Roger e Fr. Max hanno conservato delle impressioni incancellabili. Quant'è buono il Signore di permetterci di ritrovarla se non altro per lettera e di poterle dire che la giovane comunità di Taizé, in pochi anni, è cresciuta di 35 fratelli, chiamati al servizio di Colui che vuole che il mondo sia pieno della Sua conoscenza. Tutti i fratelli, dopo il noviziato, vengono mandati un po' ovunque per vivere come discepoli di Cristo. La settimana dell'unità è sempre così benefica! Quante preghiere salgono a Dio! E quanto ci auguriamo, noi, che i cristiani siano uniti in Cristo perché venga il suo Regno!

Mio figlio mi incarica di ringraziarla infinitamente della sua lettera e per l'opuscolo di sr. Maria Gabriella.

Spero che al suo ritorno le scriverà lui personalmente, ma non ho potuto resistere alla gioia di dirLe che Lei non si era ingannata e che noi Le diciamo ancora "Grazie" per l'accoglienza così straordinaria di cui siamo stati oggetto da parte Sua.

II 3 aprile 1957, ricevuto il libro su sr. Gabriella, Amélie ringrazia e racconta qualcosa sulle origini di Taizé:

La Sua buonissima lettera e il Suo magnifico libro mi sono arrivati a Cologny. Come ringraziarLa e dirLe la mia riconoscenza! Sono così commossa di tutto ciò che mi dice, sono anche profondamente toccata leggendo la vita della Sua ammirevole sorella Maria Gabriella! Che

19 Del carteggio sono perdute le risposte di madre Pia, restano solo le lettere di Amélie, che fanno parte del dossier per la beatificazione di sr. Gabriella (1983), in quanto dimostrano fino a che punto l'offerta di una vita può portare frutto. AV.

20 M. CARPINELLO, Ecumenismo in monastero, in l' Osservatore Romano,17 gennaio 2012, p. 4.

Paralellus, Recife, v. 8, n. 18, mai./ago. 2017, p. 237-250 
anima ricca e totalmente data al Salvatore, alla causa dell'ecumenismo!

(...) Dato che trovo in Lei, cara Sorella, una cara Amica, devo raccontarLe una cosa straordinaria.

Quando mio figlio ha sentito che Dio lo chiamava è partito, ha cercato il luogo dove fissarsi e fu Taizé, presso Cluny, in un castello che ha acquistato dai signori de Brie. Attualmente la signora de Brie ha avuto molte disgrazie; io non la conoscevo, ma a partire da quell'epoca è diventata un'Amica e noi la invitiamo spesso a Taizé, da me.

La signora de Brie aveva un'amica con la quale corrispondeva spesso e che era suora domenicana o forse carmelitana ${ }^{21}$.

Questa suora le scrisse questo:

Non resisto al pensiero che mi è venuto in cuore, di raccontarle un sogno. Non è che un sogno... ma è così bello ${ }^{22}$. Noi leggiamo in questo momento la storia religiosa della Francia di Georges Goyau. In questi giorni siamo all'età d'oro di Cluny e molto spontaneamente penso a voi che abitate una terra cluniacense. Ora, questa notte venivo trasportata a Taizé (che conosco tramite una cartolina mandata poco fa da mia sorella Thilde). Là si trovavano dei monaci che cantavano l'ufficio divino. La mia anima fu inondata di gioia. Poiché chiesi il nome del Padre Abate, giovane monaco dalla chioma bionda, gli occhi blu, di statura piccola, chiesi di essergli presentata...Ma, mentre avanzava verso di me, il sogno finì. Peccato! Svegliandomi l'ho terminato... Chissà mai che in un avvenire più o meno lontano uno dei suoi pronipoti non riannodi la catena del passato e ricominci Cluny! Chi conosce i disegni di Dio?

Questo è il racconto che le confido. Che ne pensa? Dio non avrà forse rivelato a questa suora ciò che preparava senza che noi lo potessimo immaginare? Com'è bello!

Poiché la signora de Brie aveva conservato questa lettera, vedendo cosa stava avvenendo a Taizé, l'ha data a mio figlio, come preziosa reliquia. Questa lettera è in data 25 gennaio 1923 e firmata sr. Hildegarde M. Loicat osb.

Come può capire, io non posso che benedire Dio e benedirlo incessantemente per il fatto che mi permette di vedere tante cose meravigliose, in un mondo che ha tanto bisogno di Lui, e direi, in un mondo che ha bisogno di soprannaturale e di vita spirituale.

Spero di rivedere mio figlio la prossima settimana; con quale gioia gli leggerò la Sua lettera così preziosa e gli parlerò di Lei, mia amica invisibile.

Pochi giorni dopo 23 :

Mia carissima e grande Amica,

con quale gioia ho ricevuto la Sua lettera così bella e quanto La ringrazio! Mi è dolce chiamarLa Amica mia, ne sono molto commossa.

${ }^{21}$ Era benedettina: il suo nome compare poi nella stessa lettera con la sigla osb.

22 Episodio riportato da K. SPINK in Frère Roger fondatore di Taizé, Bologna, 1987, p. 33 e J. M. PAUPERT, Taizé e la Chiesa di domani, Borla, Torino, p. 40.

23 il 12 aprile 1957.

Paralellus, Recife, v. 8, n. 18, mai./ago. 2017, p. 237-250 
(...)Mio figlio non ha potuto venire, come avrebbe sperato, e tra qualche giorno penso di rientrare in Francia, ma, se Dio me lo permette, spero con tutto il cuore di venirLa a trovare a fine maggio o in giugno e allora mio figlio mi accompagnerà. La ringrazio per l'affezione che porta al mio caro figlio. Sento che il Suo cuore materno così sensibile ha dovuto - accanto alle gioie celesti - soffrire della malattia della Sua ammirevole giovane suor Maria Gabriella.

Noi la vediamo in Cielo, nella gioia degli eletti e al servizio del Signore. La settimana santa che si compie a Pasqua, alla resurrezione del Salvatore, è molto impressionante per i credenti. II Salvatore ci chiede di pregare doppiamente gli uni per gli altri e per tutti coloro che non credono ancora. Noi vorremmo tanto, vero? che il suo Regno venisse. La ringrazio del posto che Lei riserva nelle sue preghiere al Priore di Taizé, come anche ai fratelli di Taizé; il compito è grande per tutti, ma specialmente per mio figlio.

II 4 maggio 1957:

Sono rientrata a Taizé 15 giorni or sono e i miei pensieri sono andati tutti i giorni a Lei. Ho avuto molte visite, ho ricevuto molta gente, il che spiega il mio silenzio. II mattino di Pasqua alle 5 e 30 eravamo riuniti in gran numero nella piccola chiesa di Taizé: due fratelli facevano la loro professione. Che gloria questo mattino di Pasqua in cui Cristo era presente, in cui tutto cantava la Sua resurrezione! In questa piccola chiesa dell'undicesimo secolo quante persone di Cluny sono venute a pregare e quanti cristiani! Non ho ancora potuto parlare un po' a lungo con mio figlio, deve ricevere tante persone! Ma lui pensa a Lei, Amica cara, con riconoscenza e affetto.

II 22 maggio 1957 Amélie ritorna sulle proprie giornate a Taizé:

... A Taizé la mia vita è molto carica e la corrispondenza ne soffre un po' (...) Lei dovrebbe avere ricevuto un libretto che ho chiesto a Fra Jean-Pierre di mandarLe: è la Regola di Taizé: ho pensato che potrebbe interessarLe (...) Le assicuro che il Priore è spesso obbligato a vivere una vita al di sopra delle forze umane (...)

Nuovamente il 6 agosto 1957:

La sua lettera meravigliosa avrebbe meritato una pronta e sollecita risposta, ma la vita a Taizé, piena di imprevisti, il periodo delle vacanze in cui accolgo anche figli e nipoti sono, stati un ostacolo per la mia corrispondenza!(...)

D'estate i visitatori e le persone che fanno ritiro sono numerosi. Pensi, cara Amica, che ho avuto la gioia, in questi giorni, di ricevere una suora benedettina e due suore domenicane, tutte e tre altrettanto meravigliose: guardandole, pensavo a Lei, (Roger si rallegra di stringere amicizia con molte religiose che vengono), ma lei è la prima Sorella con cui si è creato questo vincolo di preghiera. 
...Com'è vero tutto quello che Lei esprime così bene nella Sua lettera: "Si cerca di comprendersi e questa buona volontà è carità vera, pace, seme di unione".

\section{il 17 dicembre 1957, da Cologny:}

Le assicuro che malgrado la distanza il nostro legame di preghiera rimane molto solido e vivo. La grande forza del cristiano si trova, come lei sa benissimo, nella preghiera.

In primavera riprende la cronaca degli avvenimenti comunitari24:

(...) A Taizé la vita continua ad essere molto intensa, interessante ed arricchente dal punto di vista spirituale. Mio figlio ha ricevuto la visita di padre Villain quando avevo appena ricevuto la Sua preziosa missiva, ho potuto così parlargli di Lei. Lui La conosce, mia carissima Amica e Sorella! Noi godiamo che questo sant'uomo venga a visitarci. Fa tanto per l'avvicinamento delle Chiese. La comunità continua il suo lavoro, parecchi fratelli sono entrati nel lavoro di Dio. Le responsabilità del priore in particolare, sono sempre più grandi (...) II mio caro figlio e Fr. Max mi incaricano di dirLe quanto li abbiano commossi i Suoi messaggi e Le mandano i loro pensieri più belli in Cristo.

II 25 gennaio 1959, madre Pia riceve la notizia che, chiudendo la settimana di preghiera per l'unità dei cristiani a S. Paolo Fuori le Mura, Giovanni XXIII ha annunciato l'indizione di un Concilio ecumenico per la Chiesa universale. Pochi giorni dopo, il 3 febbraio, riceve l'ultima lettera di Amélie:

Noi abbiamo vissuto mesi straordinari dal punto di vista ecumenico. Mio figlio e fr. Max si sono recati a Roma e hanno assistito a S. Pietro all'incoronazione del papa Giovanni XXIII, che io stessa ho seguito per televisione.

In questi ultimi giorni, per terminare la settimana di preghiere per l'Unità, abbiamo avuto una grandissima emozione sentendo, domenica sera, l'annuncio di questo concilio straordinario nella sua ispirazione e nella futura realizzazione.

Penso che la cristianità vedrà belle cose e che nostro Signore, nella sua bontà, esaudisca tutte le preghiere dei suoi figli.

Noi non possiamo prevedere chi sarà convocato a questa immensa riunione, mio figlio sarà invitato come teologo e così anche fr. Max?

L'essenziale è che questo grande movimento avanzi il più presto possibile. Non sarà certamente facile. II mondo dei credenti deve prendere coscienza delle sue responsabilità di fronte all'ateismo, di fronte al male.Che lavoro immenso ha dunque la Chiesa!

Noi possiamo pregare e senza stancarci: è un dovere e un privilegio.

241 maggio 1958.

Paralellus, Recife, v. 8, n. 18, mai./ago. 2017, p. 237-250 
In questo angolino della Francia io penso a Lei, Amica così buona e cara, e La so tutta data alla preghiera, felice di tutte le bellezze della vita spirituale e, avendoLa ritrovata, Le dico tutta la mia affezione nel Signore. Ho anche per Lei i più bei pensieri da trasmetterLe da parte dei due Fratelli che Lei conosce.

A distanza di quasi un cinquantennio dalla prima visita a Grottaferrata, frère Roger rilascerà una breve intervista su madre Pia al trappista Pearce Cusack ${ }^{25}$ :

\begin{abstract}
Eravamo impressionati dalla sua capacità, malgrado fosse una reclusa, di cogliere quello che noi solo oggi comprendiamo totalmente, di parlarci della sua speranza di riconciliazione, della sua fiducia nell'opera della sua comunità. Sapeva così bene costruire su tanto poco. La sua grande venerazione per suor Gabriella e l'offerta di lei per l'Unità. Nessuno osava sperarci a quell'epoca.
\end{abstract}

Come accennato in apertura, considerato l'iter partito dall'allora trappa di Grottaferrata (oggi Nostra Signora di S. Giuseppe in Vitorchiano) meglio si comprende lo snodo di fatti che, dopo la chiusura del Vaticano II, concorre a rendere diretto il confronto fra confessioni cristiane.

Un altro avvenimento si profila parallelo a tali sviluppi, benché avvenga su un piano non esplicitamente ecumenico: la proclamazione di san Benedetto primo patrono d'Europa da parte di Paolo VI nel $1964^{26}$.

Durante gli incontri con monaci di realtà separate il giovane pro segretario Montini ha verificato ciò che Couturier ha voluto esprimere con la celebre espressione monastero invisibile, assegnata al movimento ecumenico: che la Chiesa vive i principi del suo essere una specialmente nei monasteri e che in alcuni monasteri sono di fatto già affrancate quelle barriere confessionali dolorosamente innalzatesi fra cristiani a partire dal 1517. Divenuto papa, è anche al potenziale ecumenico intrinseco al monachesimo che Montini ha inteso dare riconoscimento e propulsione affidando a san Benedetto il patrocinio del continente europeo?

25 P.CUSACK Blessed Gabriella of Unity. A Patron for the Ecumenical Movement, Cistercian Press, Roscrea, Ireland, 1995, p. 149.

${ }^{26}$ Lettera apostolica PACIS NUNTIUS, 24 ottobre 1964. 


\section{REFERENZE}

A. de VOGÜÉ. II monastero e la Chiesa, in La regola di S. Benedetto. Commento dottrinale e spirituale. Padova, 1984.

B. MARTELET. Una vita per l'unità dei cristiani, Suor Maria Gabriella. Roma, 1987.

C. TESTORE. Suor Maria Gabriella Trappista - 1914-1939. Vitorchiano, 1958.

D. SPANU. Artefice di unità: profilo spirituale della beata Maria Gabriella Sagheddu. Firenze, 2009. Cagliari, 2014.

Beata Maria Gabriella Sagheddu, testimone credibile del Vangelo di unità.

. Temi di vita spirituale nelle lettere della Beata Maria Gabriella Sagheddu. Cagliari, 2007.

E. FRANCIA. Lettere e scritti di Madre Pia. Editrice "Messa degli artisti": Roma, 1971.

G. L. PRESTIGE. Dio nel pensiero dei Padri. 1952.

GABRIELLA DELL' UNITÀ (Beata Maria Gabriella Sagheddu). Lettere dalla Trappa, a cura di M. CARPINELLO, Cinisello Balsano, 2006.

J. M. PAUPERT. Taizé e la Chiesa di domani. Borla: Torino, p. 40.

K. SPINK. Frère Roger fondatore di Taizé. Bologna, 1987, p. 33.

LEONE XIII. Bolla Apostolicae Curae. Città del Vaticano, 1896.

M. A. TESCARI. Madre Pia Gullini, una figura profetica del monachesimo italiano del XX secolo. Cantagalli, 2016.

M. A. TESCARI. Madre Pia Gullini, una grande badessa del XX secolo. Velar, 2009.

M. CARPINELLO. Ecumenismo in monastero, in l' Osservatore Romano,17 gennaio 2012 , p. 4.

Monachesimo femminile e profezia, l'azione ecumenica di Madre Maria Pia Gullini. Cantagalli, 2015.

M. DELLA VOLPE. La strada della gratitudine - Suor Maria Gabriella, Milano, 1983.

M. G. DORE; I. GIORDANI. Suor Maria Gabriella (1914-1939): dalla Trappa per l'unità della Chiesa. Brescia, 1940.

M. VILLAIN. L'Abbé Paul Couturier, apôtre de I'Unité chrétienne. Paris-Tournai, 1957.

P. CUSACK. Blessed Gabriella of Unity. A Patron for the Ecumenical Movement.

Cistercian Press: Roscrea, Ireland, 1995, p. 149. 
P.BELTRAME QUATTROCCHI. La Beata Maria Gabriella dell'Unità. Vitorchiano, 1980.

PAULO VI. Lettera apostolica PACIS NUNTIUS, 24 ottobre 1964.

R. TADINI. Ecumenismo preconciliare. Appunti-ricordo. 18-25 settembre 1947. Conservato nell'archivio del monastero di Vitorchiano.

SUOR FARA. Visita di dom Benedict Ley di Nashdom Abbey alla Trappa di Grottaferrata - 18-25 settembre 1947. Conservato nell'archivio del monastero di Vitorchiano. 